\begin{tabular}{|c|c|c|}
\hline \multicolumn{3}{|c|}{ DJS Vol. 39 (2018) 1-8 } \\
\hline 1969 & $\begin{array}{c}\text { Delta Journal of Science } \\
\text { Available online at } \\
\text { https://djs.journals.ekb.eg/ }\end{array}$ \\
\hline Research Article & & \\
\hline
\end{tabular}

\title{
On the multistage homotopy procedure for a fractional Lorenz system
}

\author{
A.R. El-Namoury1, M.M. Hikal2, Mahmoud A. Abu Ibrahim1*
}

1. Mathematics Department, Faculty of Science, Tanta University, Tanta 31527, Egypt

2. Physics and Engineering Mathematics Department, Faculty of Engineering, Tanta University, Tanta,

Egypt

* Correspondence: Mahmoud A. Abu Ibrahim; Mathematics Department, Faculty of Science, Tanta

University, Tanta 31527,Egypt; Email: Mahmoud_abuibrahim@science.tanta.edu.eg

\section{Key words:}

Fractional calculus;

Lorenz system;

The multistage

homotopy

perturbation

method.
Abstract:

In this article, the multistage homotopy perturbation method (MHPM) is applied for solving differential systems with fractional order derivatives in the Caputo sense. This method is a modification of the standard homotopy perturbation method (HPM). A fractional Lorenz system as an application is presented for which some numerical comparisons between the (MHPM) and (HPM) with the 4th order Runge-Kutta method (RK4). The results reveal that the used pre-mentioned procedure (MHPM) is a reliable and an effective tool for constructing an accurate approximate solution for the fractional Lorenz system.

Introduction:

\section{Introduction}

It is well-known that the Lorenz system [1] which is an idealized model describing turbulent flow in the atmosphere. The atmosphere is just one of many hydrodynamical systems which exhibit a variety of solution behavior: some flows are steady, others oscillate between two or more states and still others vary in an irregular manner. This last class of behavior in a fluid is known as turbulence or in more general systems as chaos. Briefly, the original derivation of the model can be described as in [2]. A two-dimensional fluid cell is heated from below and cooled from above and the resulting convective motion is modeled by a system of ordinary differential equations as:

(1)

where $x, y$ and $z$ are respectively, the variable $x$ measures the convective velocity, the variable $y$ measures the horizontal temperature variation and $z$ represents the temperature difference between rising and falling flows. $\sigma, b$ and the so-called bifurcation parameter $r$ are positive real parameters. $\sigma$ is proportional to Prandtl number (it involves the viscosity and thermal conductivity of the fluid), $r$ is a control 
parameter (it is proportional to the Rayleigh number) and $b$ involves the coefficients of thermal expansion. These equations (1) led Lorenz to the discovery of sensitive dependence of initial conditions as an essential factor of unpredictability in many systems. Since that time, about 1963, the Lorenz system has become one of the most widely studied systems of ODES because of its wide range of behavior.

Most of nonlinear systems do not have exact analytic solutions, so numerical and analytic approximation techniques must be used. Such as, 4-th order Runge-Kutta method, variation iteration method [3, 4], Adomian decomposition method (ADM) $[4,5,6]$, and the homotopy perturbation method (HPM) [7, 8]. The ADM can be applied to

solve many linear or nonlinear differential equations. But one of its disadvantages is the inherent difficulty in calculating the Adomian polynomials. (HPM) overcomes the disadvantages of (ADM). The (HPM) was first proposed by Chinese mathematician $\mathrm{He}$ in (1998) [9]. The main idea of this method is to introduce a homotopy parameter $p, p \in[0,1]$. The perturbation methods have some limitations; for example, the approximate solution involves series of small parameters which poses difficulty since most of nonlinear problems have no small parameters at all. In contrast to the traditional perturbation methods, the (HPM) technique does not require a small parameter in an equation. Moreover, there is no need to the discretization of the variables. Using the method, we can easily get an analytical approximate solution to a wide range of nonlinear problems in applied sciences. In [10], the authors presented an algorithm based on (HPM) for solving a boundary-value problem, Gill et al. [11] studied the ion-acoustic solitons in a weakly relativistic electronpositronion plasma using (HPM), In [12], an inverse problem of diffusion equation was dealt with (HPM). The HPM yields a very rapid convergence of the solution series in most cases, usually only a few iterations leading to very accurate solutions.

The pre-described model had been investigated before by many authors. Some authors studied the behavior of its solutions like [13, 14]. The lorenz system has been solved by the (ADM) [5]. Also, a fractional Lorenz system is dealt by the homotopy analysis method [15]. The model (1) with integer order derivatives was treated analytically by the (MHPM) method [16].

In this paper we apply the multistage homotopy perturbation method (MHPM) to solve Lorenz system with fractional order derivatives in the Caputo sense. Fractional derivatives play key role in modeling. Thus, Fractional Lorenz system may be introduced to provide a good simulation for the model. The (MHPM) is considered as a modification of the standard (HPM) [11]. In view of (MHPM), the (HPM) is treated as an algorithm in a sequence of subintervals for finding an accurate approximate solution to the corresponding Lorenz system.

This paper is organized as follows: Section (2) is concerned with some definitions for the fractional calculus, Section (3) is devoted to the basic ideas of the (HPM) and (MHPM). In section (4), an application for the used technique with some comparisons are introduced.

\section{Preliminaries}

In this section we review some basic definitions in fractional calculus, and some of its properties [17].

Definition 1. The function $f(x)$ is said to be in the space $L_{1}[a, b]$ if $f(x)$ is Lebesgue integrable on $[a, b]$.

Definition 2. The Riemann-Liouville fractional integral operator of order $\alpha \geq 0$ of a function $f(x)$ $\in L_{1}$ is defined by

$$
I_{a}^{\alpha} f(x)=\frac{1}{\Gamma(\alpha)} \int_{a}^{x}(x-t)^{\alpha-1} f(t) d t
$$

When $a=0$, we can write $I_{a}^{\alpha} \rightarrow I^{\alpha}$. Some properties of the operator $I_{a}^{\alpha}$ can be found in [18].

For $f(x) \in L_{1}, \alpha, \beta \geq 0$ and $\gamma>-1$; we have

$$
\begin{aligned}
& \text { 1. } I_{a}^{\alpha} I_{a}^{\beta} f(x)=I_{a}^{\beta} I_{a}^{\alpha} f(x) \\
& \text { 2. } I_{a}^{\alpha} I_{a}^{\beta} f(x)=I_{a}^{\alpha+\beta} f(x) \\
& \text { 3. } I_{a}^{\alpha} x^{\gamma}=\frac{\Gamma(\gamma+1)}{\Gamma(\gamma+\alpha+1)} x^{\alpha+\gamma}
\end{aligned}
$$

Definition 3. The fractional derivative of order $\alpha(m-1<\alpha \leq m)$ in the Caputo sense for a function $f(x) \in L_{1}$ is defined as 


$$
\begin{aligned}
D_{a}^{\alpha} f(x) & =I^{m-\alpha} \frac{d^{m}}{d x^{m}} f(x) \\
& =\frac{1}{\Gamma(m-\alpha)} \int_{a}^{x}(x-t)^{m-\alpha-1} \frac{d^{m}}{d t^{m}} f(t)
\end{aligned}
$$

Lemma 1 For $\alpha \in(m-1, m), m \in N$ and $f(x) \in$ $L_{1}$, then

- $D_{a}^{\alpha} I_{a}^{\alpha} f(x)=f(x)$

- $I_{a}^{\alpha} D_{a}^{\alpha} f(x)=f(x)-\sum_{k=0}^{m} \frac{f^{k}(a)}{k !}(x-a)^{k}$

\section{The basic idea of the HPM}

In reviewing the basic idea of the (HPM) [19]. Consider a nonlinear differential equation

$$
L(u)+N(u)=f(r), \quad r \in \Omega
$$

with boundary conditions

$$
\mathbb{B}\left(u, \frac{\partial u}{\partial n}\right)=0, \quad r \in \mathrm{T}
$$

where $L$ is a linear operator, while $N$ is a nonlinear operator, B is a boundary operator, $\Gamma$ is the boundary of the domain $\Omega$ and $f(r)$ is a known analytic function.

Using the homotopy technique proposed by Liao in [20,21], we construct a homotopy of Eq. (2) $v(r, p): \Omega \times[0,1] \rightarrow \mathrm{R}$ which satisfies

$$
H(v, p)=(1-p)\left[L(v)-L\left(u_{0}\right)\right]+p[L(v)+N(v)-f(r)]=0
$$

and which is equivalent to

$$
H(v, p)=L(v)-L\left(u_{0}\right)+p L\left(u_{0}\right)+p[N(v)-f(r)]=0
$$

where $p \in[0,1]$ is an embedding parameter and $u_{0}$ is an initial approximation of Eq. (2) which satisfies the boundary conditions (3). It is clear that Eqs. (4), (5) give

$$
\begin{aligned}
& H(v, 0)=L(v)-L\left(u_{0}\right)=0 \\
& H(v, 1)=L(v)+N(v)-f(r)=0
\end{aligned}
$$

The changing process of $p$ from zero to unity is just that of $v(r, p)$ from $u_{0}(r)$ to $u(r)$. In topology, this is called deformation, and $L(v)-L\left(u_{0}\right)$, $L(v)+N(v)-f(r)$ are called homotopic. The (HPM) assumes that the solution of Eq. (5) can be expressed as a power series in $p$;

$v=v_{0}+p v_{1}+p^{2} v_{2}+p^{3} v_{3}+\ldots$, when $p \rightarrow 1$, Eq. (5) corresponds to Eq. (4) and then Eq. (8) becomes the approximate solution of Eq. (2), i.e,

$$
u=\lim _{p \rightarrow 1} v=v_{0}+v_{1}+v_{2}+v_{3}+\ldots
$$

For the convergence of the series (9), see [7, 8]. It is worth mentioning that the operator $L$, and the initial approximation are freely chosen.

\subsection{The (HPM) for a system of fractional differential equations}

In this section, we extend the application of (HPM) to solve a system of fractional order differential equations of the form:

$D^{\alpha i} y_{i}=f_{i}\left(t, y_{1}, y_{2}, \cdots, y_{n}\right)$

with the initial condition:

$$
y_{i}^{(k)}\left(t_{0}\right)=c_{i}^{k}, \quad k=0,1,2, \cdots, m_{i}
$$

where $f_{i}$ 's are linear or nonlinear functions and

$m_{i}<\alpha_{i} \leq m_{i}+1, \quad m_{i}=0,1,2, \cdots$

Following the approach given for the (HPM) in $[7,8]$, we construct a homotopy for the Eq. (10) which satisfies the following relations:

$D^{\alpha i} y_{i}=p f_{i}\left(t, y_{1}, y_{2}, \cdots, y_{n}\right)$

where $i=1,2, \cdots, n, p \in[0,1]$. As mentioned before, the basic assumption is that the solution of Eq. (10) can be expanded as a power series in $p$ as:

$y_{i}(t)=y_{i 0}+p y_{i 1}+p^{2} y_{i 2}+\ldots$

and the initial conditions can be written as

$y_{i 0}^{(k)}\left(t_{0}\right)=y_{i}^{(k)}\left(t_{0}\right)=c_{i}^{k}, \quad y_{i j}^{(k)}\left(t_{0}\right)=0 \quad j=1,2,3, \ldots$

The nonlinear functions $f_{i}$ 's can be decomposed as

$f_{i}=\sum_{n=0}^{\infty} p^{n} H_{i n}$

where $H_{\text {in }}$ 's are called He polynomials that defined by [22] as

$H_{\text {in }}=\frac{1}{n !} \frac{d^{n}}{d \lambda^{n}}\left[f_{i}\left(\sum_{j=0}^{n} \lambda^{j} y_{1 j}, \sum_{j=0}^{n} \lambda^{j} y_{2 j}, \cdots, \sum_{j=0}^{n} \lambda^{j} y_{n j}\right)\right]_{\lambda=0}$ 
Substituting Eq. (12) and Eq. (14) into Eq. (11) and collecting the terms of the same powers of $p$, we obtain

$p^{0}: \quad D^{\alpha i} y_{i 0}=0$

$p^{1}: \quad D^{\alpha i} y_{i 1}=H_{i 1}\left(t, y_{10}, y_{20}, \cdots, y_{n 0}\right)$

$p^{2}: \quad D^{\alpha i} y_{i 2}=H_{i 2}\left(t, y_{10}, y_{20}, \cdots, y_{n 0}, y_{11}, y_{21}, \cdots, y_{n 1}\right)$

$p^{3}: \quad D^{\alpha i} y_{i 3}=H_{i 3}\left(t, y_{10}, \cdots, y_{n 0}, \cdots, y_{n 1}, \cdots, y_{n 2}\right)$

Applying the integral operator $I_{t_{0}}^{\widetilde{\alpha}_{i}}\left(I_{t_{0}}^{\widetilde{\alpha}_{i}}\right.$ means integration from $t_{0}$ to $t$ ) for both sides of the Eqs. (16) with the initial conditions (13) hence, the unknown function $y_{i j}(t)$ can be determined. By setting $p=1$ in (12), the HPM serious solutions to Eq. (11) are given as

$y_{i}(t)=\sum_{j=1}^{\infty} y_{i j}(t), \quad i=1,2,3, \cdots, n$

So, the m-approximations of the (HPM) series solution can be expressed as

$y_{i}(t) \approx \sum_{j=0}^{m-1} y_{i j}(t), \quad i=1,2,3, \cdots, n$.

\subsection{The concept of the (MHPM)}

Unfortunately, (HPM) gives a good approximation only in a neighborhood of the initial time. The (MHPM) modification is introduced to improve this defect. This modification was introduced by $\mathrm{M}$. Chowdhur in (2009) [23]. According to the technique of (MHPM), the (HPM) is treated as an algorithm in a sequence of intervals for finding accurate approximate solution to Eq. (11). So, the time interval $[0 ; t)$ can be divided into a sequence of subintervals $\left[t_{0} ; t_{1}\right] ;\left[t_{1} ; t_{2}\right] ; \ldots ;\left[t_{j-1} ; t_{j}\right]$, in which $t_{0}=0, t_{j}=t$. Every subinterval can be chosen to have the same length $\Delta t$. Thus, if we denote to the initial time of every subinterval with $t^{*}$, the initial approximations take the form

$$
y_{i 0}^{k}(t)=y_{i}^{k}\left(t^{*}\right)
$$

where $t^{*}$ is the left-end point of each subinterval, $\mathrm{k}$ represents the number of the current subinterval and when $\mathrm{k}=1, \mathrm{t}^{*}=\mathrm{t}_{0}$. Therefore, the series solution in every subinterval becomes in the form

$$
y_{i}^{k}(t)=\sum_{j=0}^{\infty} y_{i j}^{k}\left(t-t^{*}\right), \quad i=1,2, \cdots, n
$$

\section{Applications}

In this section, we study the lorenz system (1) in case of replacing the first order derivative in time by the Caputo fractional derivative of order $\alpha,(0<\alpha \leq 1)$ as

$$
\begin{aligned}
D^{\alpha} x(t) & =\sigma(y-x) \\
D^{\alpha} y(t) & =r x-y-x z \\
D^{\alpha} z(t) & =x y-b z
\end{aligned}
$$

where $\mathrm{D}=\frac{d}{d t}$, subject to the initial conditions

$x\left(t^{*}\right)=a_{0}, \quad y\left(t^{*}\right)=b_{0}, \quad z\left(t^{*}\right)=c_{0}$

According to the (HPM)(back to section (3.1)), by collecting the terms of the same powers of $p$, the Eqs. (19) is turned to an infinite number of linear systems of triple fractional differential equations as follows:

$$
\begin{aligned}
p^{0}: \quad D^{\alpha} x_{0}(t) & =0 \\
D^{\alpha} y_{0}(t) & =0 \\
D^{\alpha} z_{0}(t) & =0 \\
p^{1}: \quad D^{\alpha} x_{1}(t) & =\sigma\left(y_{0}(t)-x_{0}(t)\right) \\
D^{\alpha} y_{1}(t) & =r x_{0}(t)-y_{0}(t)-x_{0}(t) z_{0}(t) \\
p^{\alpha} z_{1}(t) & =-b z_{0}(t)+x_{0}(t) y_{0}(t) \\
p^{2}: \quad D^{\alpha} x_{2}(t) & =\sigma\left(y_{1}(t)-x_{1}(t)\right) \\
D^{\alpha} y_{2}(t) & =r x_{1}(t)-y_{1}(t)-x_{0}(t) z_{1}(t)-x_{1}(t) z_{0}(t) \\
D^{\alpha} z_{2}(t) & =-b z_{1}(t)+x_{1}(t) y_{0}(t)+x_{0}(t) y_{1}(t)
\end{aligned}
$$

and so on for $p^{3}, p^{4}, \ldots$. with the initial conditions

$x_{0}(t *)=x(t *)=a_{0} ; y_{0}\left(t^{*}\right)=y\left(t^{*}\right)=b_{0} ; z_{0}\left(t^{*}\right)=z\left(t^{*}\right)=c_{0}$

$x_{i}\left(t^{*}\right)=0 ; \quad y_{i}\left(t^{*}\right)=0 ; \quad z_{i}\left(t^{*}\right)=0, i=1,2,3, \ldots$

and so on.

Now we solve the above systems (21) for the unknown $x_{\mathrm{m}}(\mathrm{t}), y_{\mathrm{m}}(\mathrm{t})$ and $z_{\mathrm{m}}(\mathrm{t})$ by applying the inverse operator $I^{\alpha},(0<\alpha \leq 1)$, where the series solutions are considered as

$x(t)=\sum_{m=0}^{\infty} x_{m}(t), y(t)=\sum_{m=0}^{\infty} y_{m}(t)$ and $z(t)=\sum_{m=0}^{\infty} z_{m}(t)$. 
Therefore, the $\mathrm{m} t h$ term approximation for the solutions of (19) can be expressed as

$$
\begin{aligned}
x_{m}(t) & =a_{m} \frac{\left(t-t^{*}\right)^{m \alpha}}{\Gamma(m \alpha+1)}, \\
y_{m}(t) & =b_{m} \frac{\left(t-t^{*}\right)^{m \alpha}}{\Gamma(m \alpha+1)}, \\
z_{m}(t) & =c_{m} \frac{\left(t-t^{*}\right)^{m \alpha}}{\Gamma(m \alpha+1)}
\end{aligned}
$$

where the coefficients take the form

$$
\begin{aligned}
& a_{m}=\sigma\left(b_{m-1}-a_{m-1}\right), \\
& b_{m}=r a_{m-1}-b_{m-1}-(m-1) ! \Sigma_{k=0}^{m-1} \frac{a_{k} c_{m-1-k}}{k !(m-1-k) !}, \\
& c_{m}=-b c_{m-1}+(m-1) ! \Sigma_{k=0}^{m-1} \frac{a_{k} b_{m-1-k}}{k !(m-1-k) !}
\end{aligned}
$$

To carry out the used technique in every subinterval of equal length $\Delta \mathrm{t},\left[0 ; t_{1}\right) ;\left[t_{1} ; t_{2}\right)$; $\left[t_{2} ; t_{3}\right) ; \ldots\left[t_{\mathrm{n}-1} ; t\right)$, we need to know the values of the following initial conditions for every subinterval:

$a_{0}=x\left(t^{*}\right), \quad b_{0}=y\left(t^{*}\right), \quad c_{0}=z\left(t^{*}\right)$

In general, we don't have these information at our clearance except at the initial point $t^{*}=t_{0}=$ 0 but we can obtain these values by using the (MHPM) as given in Section (3.2).
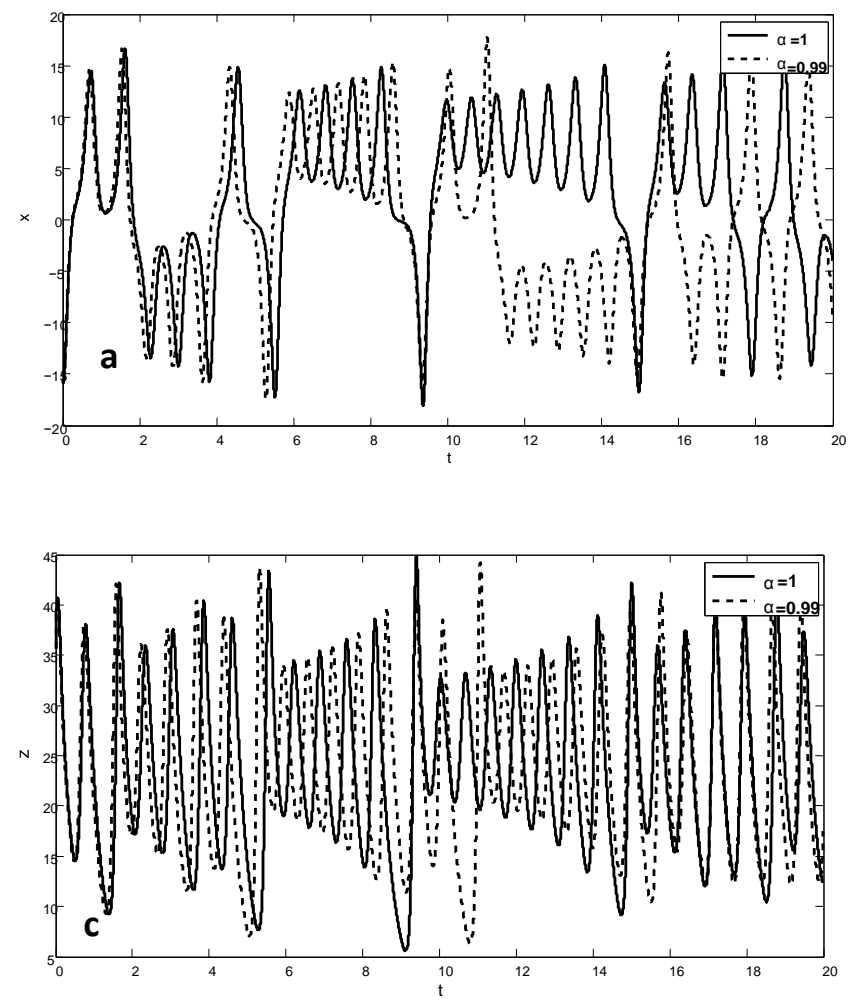

\subsection{Results and discussion}

Following the studies of Lorenz, he chose $\sigma=$ 10 (a realistic value for water) and $b=8 / 3$. For comparison with Chowdhury et al. [23], we take the initial conditions as $x(0)=-15.8, y(0)=$ 17.48 and $z(0)=35.64$ at the standard case $(\alpha=$ $1)$. For the system (1), it is well known that the value $\mathrm{r}=\frac{\sigma(\sigma+b+3)}{\sigma-b-1}$ is critical value for the chaos sets $[1,2]$. So, in this case the critical value is $r=24.74$. Thus, for comparison, we shall consider two cases: $r=28$ where the system exhibits chaotic behavior and $r=23.5$ where the system is non-chaotic.

\subsubsection{Chaotic solutions}

At first, we consider the chaotic case when $\mathrm{r}=$ 28 with the parameters $\sigma$ and $b$ as given above. The (MHPM)-series solutions of chaotic system (19) for t with $x(t), y(t)$ and $z(t)$ when $\alpha$ change from 1 to 0:99 are plotted in Fig. (1). The phase portraits of $x y z$ when $\alpha=1,0.99$ and 0.97 respectively, using 10-term (MHPM) with $\Delta \mathrm{t}=$ 0.01 in the time domain $[0 ; 20]$ are obtained as presented in Fig. (2). In Fig. (2a), (2b) and (2c), the solutions agree with the solutions in [23] when $\alpha=1$. But, they are different when the derivative order $\alpha$ is changed.

In table (1), we present the absolute errors between the 10-term (MHPM) solutions and the 10-term HPM solutions and the (RK4) solutions on time step $h=0.01$ and $\alpha=1$ for $\mathrm{r}=28$.

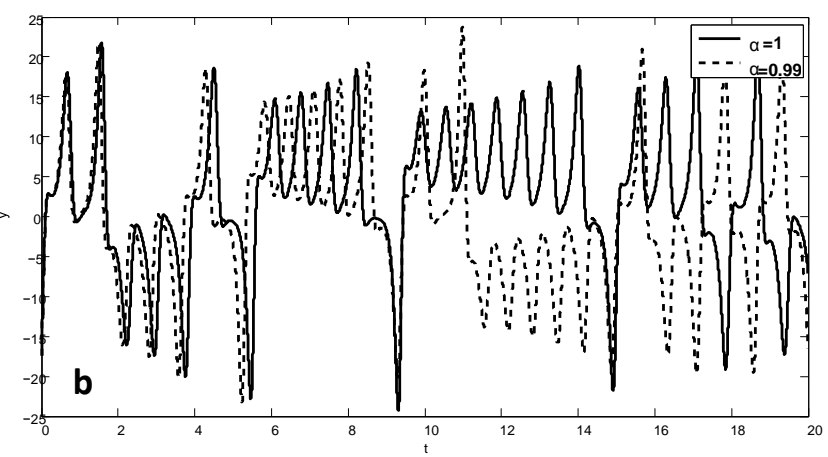

Figure (1): MHPM solutions for chaotic case of fractional Lorenz system in the standard case and when $\alpha=0.99$. (a) Time response of $x(t)$; (b) Time response of $y(t)$ and (c) Time response of $z(t)$. 

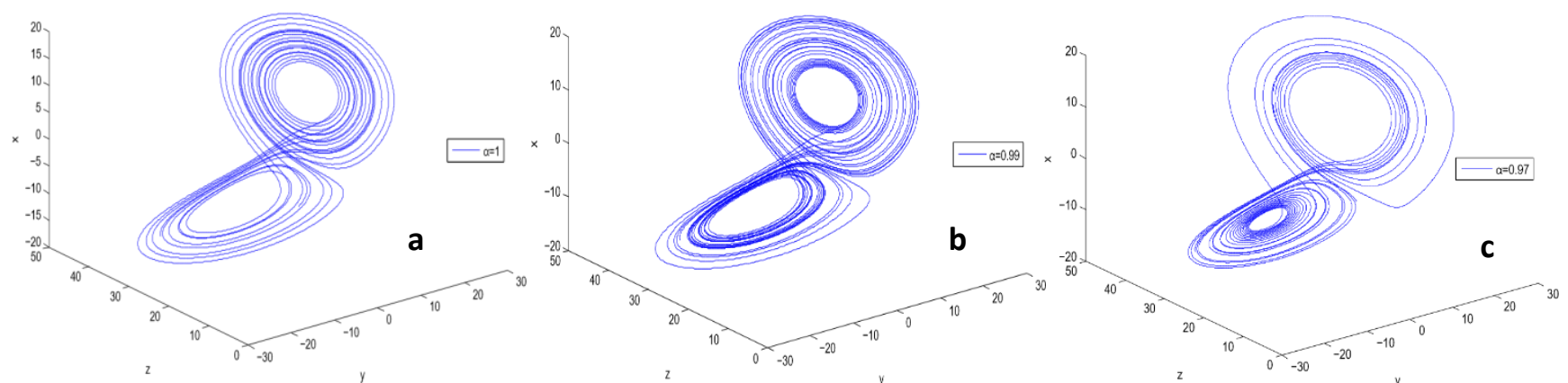

Figure (2): Phase portraits using 20-term MHPM on $\Delta t=0.01$ for $r=28$ when (a)Phase portrait for chaotic case when $\alpha=1$; (b)Phase portrait for chaotic case when $\alpha=0.99$; (c)Phase portrait for chaotic case when $\alpha=0.97$.

Table (1): Differences between 10-term HPM and 10-term MHPM with RK4 solutions for $\mathrm{r}=28, \alpha=1$

\begin{tabular}{|c|c|c|c|c|c|c|}
\hline \multirow[b]{2}{*}{$t$} & \multicolumn{3}{|c|}{$\Delta=\left|M H P M_{0.001}-R K 4_{0.01}\right|$} & \multicolumn{3}{|c|}{$\Delta=\left|H P M_{0.001}-R K 4_{0.01}\right|$} \\
\hline & $\Delta x$ & $\Delta y$ & $\Delta z$ & $\Delta x$ & $\Delta y$ & $\Delta z$ \\
\hline 1 & $3.4500 E-005$ & $2.9379 E-005$ & $1.3768 E-004$ & $1.5090 E+007$ & $6.7601 E+006$ & $4.5031 E+007$ \\
\hline 2 & $1.0694 E-004$ & $1.4802 E-004$ & $2.0225 E-004$ & $7.3686 E+009$ & $5.3799 E+008$ & $2.4095 E+010$ \\
\hline 3 & $8.4308 E-005$ & $4.9054 E-004$ & $7.1111 E-004$ & $2.7486 E+011$ & $7.3601 E+010$ & $9.2858 E+011$ \\
\hline 4 & $1.3897 E-004$ & $1.5796 E-004$ & $3.5045 E-005$ & $3.5959 E+012$ & $1.3310 E+012$ & $1.2354 E+013$ \\
\hline 5 & $4.5974 E-004$ & $7.7596 E-004$ & $4.8366 E-004$ & $2.6482 E+013$ & $1.1475 E+013$ & $9.1919 E+013$ \\
\hline 6 & $0.0031 E-000$ & $0.0052 E-000$ & $4.3641 E-004$ & $1.3554 E+014$ & $6.4540 E+013$ & $4.7371 E+014$ \\
\hline 7 & $0.0028 E-000$ & $0.0034 E-000$ & $0.0017 E-000$ & $5.3949 e+014$ & $2.7363 E+014$ & $1.8949 E+015$ \\
\hline 8 & $0.0090 E-000$ & $0.0145 E-000$ & $0.0044 E-000$ & $1.7861 E+015$ & $9.4787 E+014$ & $6.2973 E+015$ \\
\hline 9 & $0.1280 E-000$ & $0.2492 E-000$ & $0.0268 E-000$ & $5.1370 E+015$ & $2.8206 E+015$ & $1.8164 E+016$ \\
\hline
\end{tabular}

\subsubsection{Non- chaotic solutions}

Now, we consider the non-chaotic case when $\mathrm{r}=$ 23.5 with the other parameters $\sigma$ and $b$ as given before. The (MHPM)-series solutions of chaotic system (19) for $t$ with $x(t), y(t)$ and $z(t)$ when $\alpha$ change from 1 to 0.99 are plotted in Fig. (3). The phase portraits of $x y z$ when $\alpha=1 ; 0.99$ and
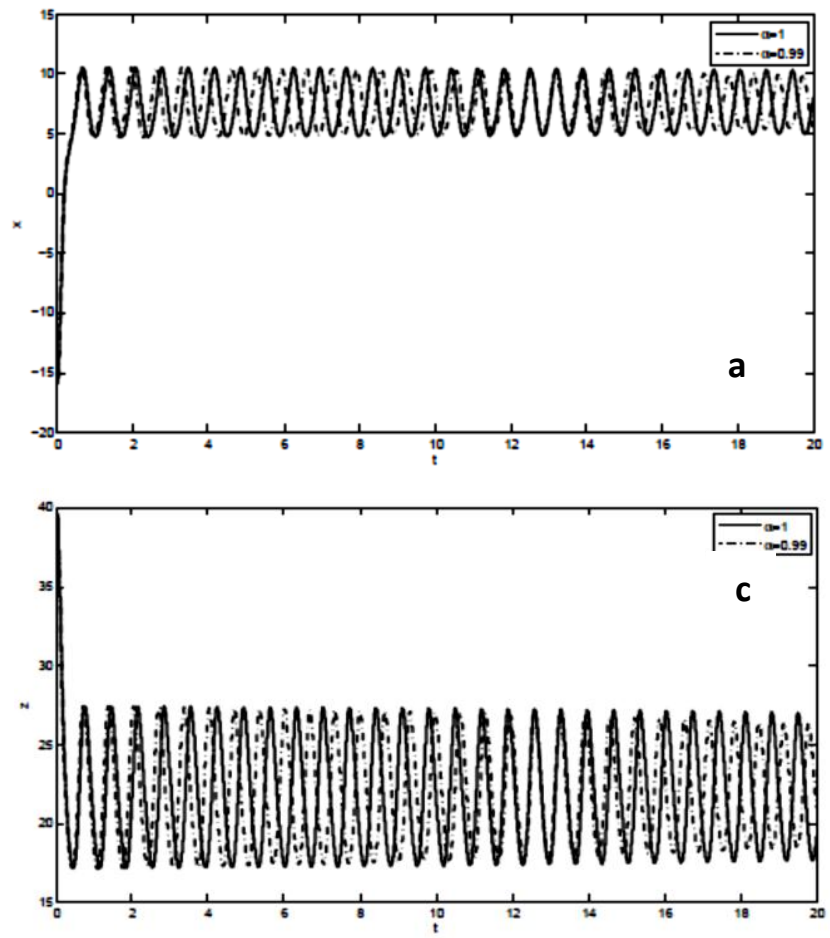

0.95, respectively, using 10-term (MHPM) with $\Delta \mathrm{t}=0.01$ in the time domain $[0,20]$ are obtained as presented in Fig. (4). In Fig. (4a) the solution agrees with (MHPM) solution in [23], while in Fig. (4b) and (4c), they are different when the derivative order $\alpha$.

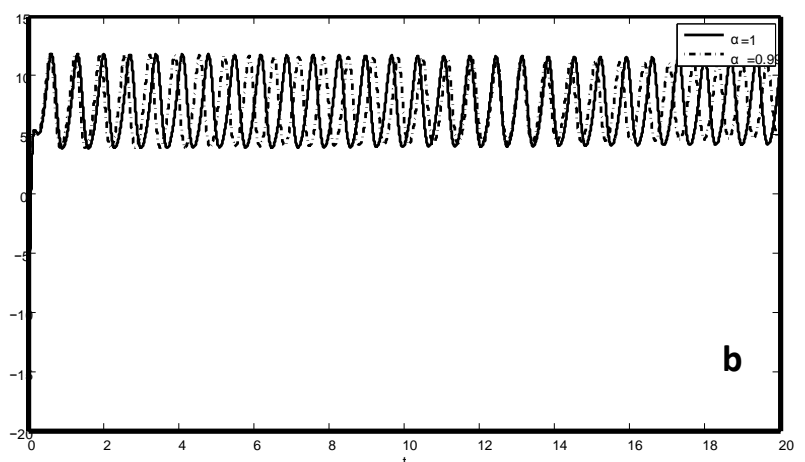

Figure (3): MHPM solutions for non-chaotic case of fractional Lorenz system when $\alpha=1,0.99$. (a) Time response of $x(t)$; (b) Time response of $y(t)$ and (c) Time response of $z(t)$. 

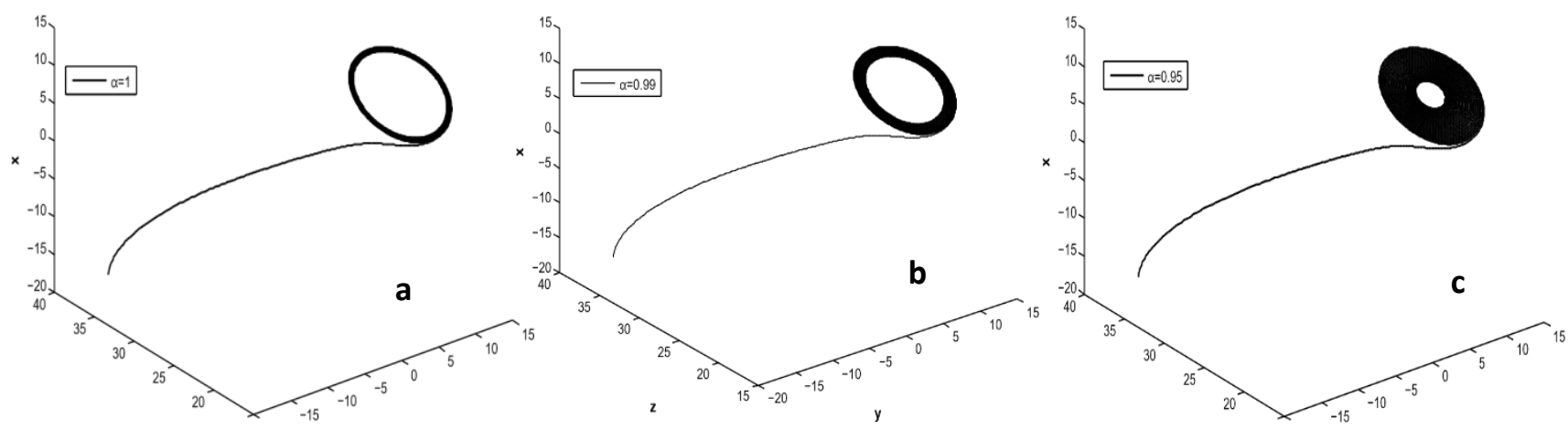

Figure (4): MHPM solutions for non chaotic cases of fractional Lorenz system when (a) $\alpha=1$; (b) $\alpha=0.99$; (c) $\alpha=0.95$.

Table (2): Differences between 10-term HPM and 10-term MHPM with RK4 solutions for $\mathrm{r}=23.5, \alpha=1$

\begin{tabular}{|c|c|c|c|c|c|c|}
\hline \multirow[b]{2}{*}{$t$} & \multicolumn{3}{|c|}{$\Delta=\left|M H P M_{0.001}-R K 4_{0.01}\right|$} & \multicolumn{3}{|c|}{$\Delta=\left|H P M_{0.001}-R K 4_{0.01}\right|$} \\
\hline & $\Delta x$ & $\Delta y$ & $\Delta z$ & $\Delta x$ & $\Delta y$ & $\Delta z$ \\
\hline 1 & $2.5671 E-006$ & $6.9147 E-006$ & $1.8169 E-005$ & $2.2745 E+007$ & $2.6192 E+007$ & $8.4856 E+007$ \\
\hline 2 & $2.6364 E-005$ & $1.0219 E-005$ & $6.2253 E-005$ & $1.0180 E+010$ & $2.0639 E+010$ & $4.2209 E+010$ \\
\hline 3 & $4.1138 E-005$ & $7.3391 E-006$ & $7.9878 E-005$ & $3.6736 E+011$ & $8.8160 E+011$ & $1.5882 E+012$ \\
\hline 4 & $5.8762 E-005$ & $7.9162 E-005$ & $5.0296 E-005$ & $4.7218 E+012$ & $1.2308 E+013$ & $2.0878 E+013$ \\
\hline 5 & $9.6981 E-005$ & $9.9348 E-005$ & $8.9802 E-005$ & $1.7470 E+014$ & $9.4183 E+013$ & $1.5423 E+014$ \\
\hline 6 & $4.8259 E-005$ & $9.8317 E-005$ & $3.8874 E-005$ & $1.7470 E+014$ & $4.9436 E+014$ & $7.9099 E+014$ \\
\hline 7 & $5.8669 E-005$ & $1.9656 E-004$ & $8.2328 E-005$ & $6.9158 E+014$ & $2.0033 E+015$ & $3.1532 E+015$ \\
\hline 8 & $9.7538 E-007$ & $7.3327 E-005$ & $1.3607 E-004$ & $2.2802 E+015$ & $6.7217 E+015$ & $1.0452 E+016$ \\
\hline 9 & $7.7121 E-005$ & $5.4564 E-005$ & $2.8032 E-004$ & $6.5366 E+015$ & $1.9533 E+016$ & $3.0087 E+016$ \\
\hline
\end{tabular}

For a comparison between HPM and (MHPM) with the numerical method (RK4) in case of the integer order $\alpha=1$ for $\mathrm{r}=23: 5$, the absolute errors between the 10-term (MHPM) solutions and the 10-term HPM solutions and the (RK4) solutions are considered in the following table (2).

\section{Conclusions}

The main aim of this work is to use the multistage homotopy perturbation method (MHPM) which is a modification to the standard (HPM) to solve the fractional Lorenz system. The numerical comparison between the solutions of (MHPM) and (HPM) with the fourth order Runge-Kutta method (RK4) tells us that the solutions obtained by the standard (HPM) aren't valid for a long time. While the solutions obtained by the (MHPM) are highly accurate for a longtime, usually only a few approximations leading to very accurate solutions. So, the (MHPM) is reliable and effective tool moreover, it is very easy to apply.

\section{References}

[1] C. Sparrow, The Lorenz equations: bifurcations, chaos, and strange attractors, Springer-Verlag, New York, 1982 .

[2] Lorenz EN., Deterministic nonperiodic ow, J. Atmospheric Sci, 20(2) (1963) 130-141.

[3] S. Momani, Non-perturbative analytical solutions of the space and time fractional Burgers equations, Chaos, Solitons and Fractals 28 (2006) 930-937.

[4] Z. Odibat and S. Momani, Approximate solutions for boundary value problems of time-fractional wave equation, Appl. Math. Comput., 181 (2006) 767-774.

[5] I. Hashim, M.S.M. Noorani, R. Ahmad, S.A. Bakar, E.S. Ismail and A.M. Zakaria, Accuracy of the Adomian decomposition method applied to the Lorenz system, Chaos, Solitons and Fractals 28 (2006) 1149-1158.

[6] S. Momani and Z. Odibat, Analytical solution of a time-fractional NavierStokes equation by Adomian decomposition method, Appl. Math. Comput. 177 (2006) 488-494.

[7] He JH, Homotopy perturbation technique, Comput. Meth. Appl. Mech. Engng., 178 (1999) 257-262. 
[8] He JH, A coupling method of homotopy technique and perturbation technique for nonlinear problems, Int. J. Non-Linear Mech., 35(1) (2000) 37-43.

[9] He JH, Approximate analytical solution of Blasius equation, Commun Nonlinear Sci. Numer. Simul., 3 (1998) 260-263.

[10] M.A. Noor and S.T. Mohyud-Din, An efficient algorithm for solving fifth-order boundary value problems, Math. Comput. Model, 45(7-8) (2007) 954-964

[11] TS. Gill, A. Singh, H. Kaur, NS. Saini and P. Bala. Ion-acoustic solitons in weakly relativistic plasma containing electron-positron and ion, Physics Letters A 361 (2007) 364-367.

[12] F. Shakeri and M. Dehghan, Inverse problem of discussion equation by Hes homotopy perturbation method, Physica Scripta, 75(4) (2007) 551-556.

[13] L. Qi, L. XiaoXin and Z. ZhiGang, Sufficient and necessary conditions for Lyapunov stability of Lorenz system and their application, SCIENCE CHINA Information Sciences, 53(8) (2010) 15741583.

[14] J. Alvarez, Synchronization in the Lorenz System: Stability and Robustness, Nonlinear Dynamics 10(1) (1996) 89-103.

[15] A.K. Alomari, M.S.M. Noorani, R. Nazar and C.P. $\mathrm{Li}$, Homotopy analysis method for solving fractional
Lorenz system, Commun. Nonlinear Sci. Numer. Simulat., 15 (2010) 1864-1872.

[16] M.S.H. Chowdhury, I. Hashim and S. Momani, The multistage homotopy-perturbation method: A powerful scheme for handling the Lorenz system, Chaos, Solitons and Fractals, 40 (2009) 1929-1937.

[17] K. S. Miller and B. Ross, An Introduction to the Fractional Calculus and Fractional Differential Equations, Wiley-Interscience, New York, 1993.

[18] I. Podlubny, Fractional Differential Equations, Academic Press, New York, 1999.

[19] He JH. Homotopy perturbation technique. Comput. Math. Appl. Mech. Eng. (1999) 178- 257.

[20] S.J. Liao, An approximate solution technique not depending on small parameters: a special example, Int. J. Non-Linear Mechanics 30 (3) (1995) 371-380.

[21] S.J. Liao, Boundary element method for general nonlinear dierential operators, Engineering Analysis with Boundary Element, 20(2) (1997) 91-99.

[22] A. Ghorbani, Beyond Adomian polynomials: He polynomials, Chaos, Solitons and Fractals 39 (2009) 1486-1492.

[23] M. Chowdhury and I. Hashim, Application of multistage homotopy-perturbation method for the solutions of the Chen system, Nonlinear Analysis: RealWorld Applications 10 (2009) 381- 391. 